\title{
Model Predictive Approaches for Active Surge Control in Centrifugal Compressors
}

\section{Journal Article}

Author(s):

Torrisi, Giampaolo; Grammatico, Sergio; Cortinovis, Andrea; Mercangöz, Mehmet; Morari, Manfred; Smith, Roy (D)

Publication date:

2017-11

Permanent link:

https://doi.org/10.3929/ethz-b-000234980

Rights / license:

In Copyright - Non-Commercial Use Permitted

Originally published in:

IEEE Transactions on Control Systems Technology 25(6), https://doi.org/10.1109/TCST.2016.2636027 


\title{
Model Predictive Approaches for Active Surge Control in Centrifugal Compressors
}

\author{
Giampaolo Torrisi, Sergio Grammatico, Andrea Cortinovis, Mehmet Mercangöz, Manfred Morari, \\ and Roy S. Smith
}

\begin{abstract}
Model predictive control (MPC) techniques are considered for industrial centrifugal compression systems with nonlinear dynamics, to address process and antisurge control for reaching the desired pressure ratio and surge distance. We consider a contractive nonlinear MPC formulation that ensures asymptotic stability of the closed-loop system by imposing the decrease of a quadratic Lyapunov function via an additional constraint. We discuss recursive feasibility and estimate the region of attraction via numerical methods. We also consider alternative MPC formulations, including offset-free linear and nonlinear MPC to handle the effects of disturbances and unmodeled dynamics. The computational efficiency of an approximation based on sequential quadratic programming (SQP), that yields a closed-loop performance comparable to the full nonlinear MPC is also discussed. All of the controllers considered are tested in simulations that emulate a realistic test bench and their computational time is assessed on an industrial Programmable Logic Controller (PLC). Their performance is compared with standard industrial control in nominal and perturbed cases replicating the typical and critical disturbances and model mismatches. The numerical results show that the SQP and nonlinear MPC methods outperform the other controllers in the considered scenarios, based on closed-loop performance metrics for the surge margin, the reference tracking accuracy, and the system actuation, without significantly increasing the computational time.
\end{abstract}

\section{INTRODUCTION}

G AS compression systems represent a key technology in process industry and gas transportation systems. Compressors increase the thermodynamic energy of a gas, in particular through pressure increase, by employing mechanical energy delivered by gas turbines or electric motors mechanically connected to the compressor shaft. The former represent a classical mechanical control setting, which is lately being frequently contested by the latter, thanks to wider flexibility, ease of operation and economic benefits [1]. Moreover, electrical variable speed drives achieve high efficiency and low time constants in regulation problems [2].

This work was supported by Marie Curie FP7-ITN through the Project entitled Energy Savings from Smart Operation of Electrical, Process and Mechanical Equipment under Contract PITN-GA-2010-264940.

G. Torrisi, M. Morari, and R. S. Smith are with the Automatic Control Laboratory, ETH Zürich, CH-8092 Zürich, Switzerland (e-mail: torrisig@ control.ee.ethz.ch; morari@control.ee.ethz.ch; rsmith@control.ee.ethz.ch).

S. Grammatico is with the Control Systems Group, Department of Electrical Engineering, Eindhoven University of Technology, NL-5612 AZ Eindhoven, The Netherlands (e-mail: s.grammatico@tue.nl).

A. Cortinovis and M. Mercangöz are with the ABB Corporate Research Center, CH-5405 Baden, Switzerland (e-mail: andrea.cortinovis@ch.abb.com; mehmet.mercangoez@ch.abb.com).
Axial and centrifugal compressors have been extensively studied in the literature. The nonlinear dynamics generates an instability, called surge, that consists of periodic oscillation of the system variables, i.e., mass flow rates, speed, and pressures. Such oscillations can result in deep surge, which has striking effects on the machine such as irreversible damage of the blades tips and bearings.

Surge phenomena have long been analyzed with bifurcation theory, considering the compressor speed as the parameter generating such bifurcations [3]-[7]. The phenomenon is even more complicated in axial compressors, since rotating stall can occur, and this adds additional stable but undesired equilibria to the system. Surge determines an unstable region in the variable space, which is usually displayed in compressor maps by an experimentally derived surge line (SL). The closer the compressor operates to such a line, the higher the risk of surge occurrence. On the other hand, external conditions, e.g., the downstream process, can move the system toward the surge region, thus forcing operations close to the surge region. Alternatively, efficiency considerations can drive the choice of the desired operating point close to the surge region, where the efficiency is typically the highest. The action of external disturbances, such as an unmodeled back pressure of the upstream and downstream processes, can then result in surge occurrence in tens of milliseconds. Hence, the control objective is to track the desired operating point guaranteeing safe operations of the compression system.

Simple but descriptive models have long been available in the literature [8]-[10]. A general compression system consists of a compressor, which takes process gas from upstream conditions and pumps it into a downstream volume (plenum) through a duct. The discharge from the plenum is via a throttle in an exit duct. Several actuators have been considered for surge avoidance, including outlet valves [11] or special closed coupled valves [12]. Unfortunately, in industry, these solutions are not possible to implement at present. The use of variable speed drives makes it possible to consider the motor torque as an actuator for the system. In [10], a PID control of the torque is considered, and in [13], a nonlinear control is designed via backstepping techniques. The structure of the model is exploited in [14] to design a Lyapunov-based controller for 
the close-coupled valve, which results in a control strategy analogous to sliding mode to move the operation toward the safe zone.

Industrial plants are nowadays equipped with recycle valves, which regulate bypass circuits connecting the outlet plenum to the compressor inlet. Moving the process gas through such circuits reduces almost instantaneously the pressure ratio and increases the mass flow through the compressor, and this results in moving the system far from the surge instability region. Due to their efficacy, critical industrial compression plants always have such systems installed. Standard state-ofthe-art control of such devices is usually decoupled from the torque control and consists of a PI scheme with feedforward control action, based on the measured distance from the surge region. The controllers are typically integrated in higher level control systems, which interface multiple compressors for load-sharing and other turbomachinery [15], [16].

The improvements in computational power of embedded controllers have recently opened the way to advanced controllers such as model predictive control (MPC) for time critical applications such as compressor control. The benefits to the industrial implementation are shown experimentally in [17], where it is observed that tuning the standard PI control for surge avoidance becomes extremely difficult in the presence of external disturbances, since a specific tuning for one scenario can be ineffective for all others. In [18], a linearized MPC is employed for a compression system, driven by a gas turbine, which controls the rotor speed and the inlet guide vane positioning. A configuration with a recycle valve is used in [19]. In [20], additional temperature limitations are considered as output constraints in the linearized MPC formulation. Dealing with nonlinear dynamical models complicates the MPC optimization problem, especially in the presence of low sampling times. Linearization at every time step is the strategy employed in [21]-[23], which is validated experimentally, addressing technical feasibility and implementability in actual industrial controllers. In [24], an explicit multiparametric nonlinear programming method is applied to the control of the outlet valve of a compression system. In [25], a controller for the recycle valve and the drive torque is designed, based on backstepping techniques combined with MPC. Additionally, the system is robust to possible mismatches on the output valve characteristics.

The nonlinearity of the compression system limits the achievable performance of linearized MPC approaches. In [26], such a strategy is compared with nonlinear MPC that considers the full nonlinear model [27]. The divergence between linear and nonlinear model predictions is considerably reduced by the sequential quadratic programming (SQP) approach, which approximates the nonlinear problem by successive quadratic programs (QPs) [28], [29]. In the real-time iteration approach presented in [30], only one QP problem is solved at every time step. A discussion on the stability and quality of this approximation is presented in [31]. Moreover, the computation time achievable with embedded software [32] makes this approach implementable in real time.

In this paper, we propose a numerical method for assessing the asymptotic stability of nonlinear MPC controllers for

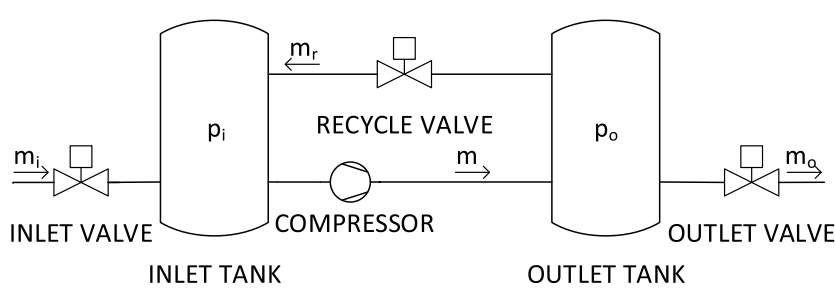

Fig. 1. Compression system diagram.

compression systems. Specifically, we apply the theory of contractive MPC [27], [33] that introduces, in the formulation, the decrease of a control Lyapunov function (CLF) as an additional constraint. We follow this approach and, as the system dynamics are nonlinear, propose to check recursive feasibility of the Lyapunov constraint via a numerical test.

Since the assumptions for ensuring closed-loop stability can be too restrictive for typical industrial plants, we also consider alternative MPC formulations for handling unmodeled disturbances and model mismatch. In particular, we apply linear and nonlinear offset-free MPC controllers [34]-[36] for tracking the desired process reference, defined as a target pressure ratio and surge distance (SD). Next, we compare all the MPC approaches considered and provide insights on the control design, with respect to both the disturbance model and the computational complexity. Finally, we show the numerical results in closed-loop simulations representing a realistic test bench, and assess all the proposed methods with respect to the current industrial practice.

The paper is organized as follows. In Section II, the model of the compression system from [22] is considered, based on the Greitzer equations, and the state-of-the-art industrial control is introduced. In Section III, the contractive nonlinear MPC problem is discussed, along with the numerical analysis for stability and recursive feasibility. Section IV discusses the offset-free MPC formulations, and in Section V, the performance of the controllers is numerically simulated, first in the nominal case, and then considering additional disturbances and parameter mismatch. Section VI concludes this paper and provides an outlook for industrial exploitation.

\section{General Plant For Industrial COMPRESSION SYSTEMS}

A general compression system with a recycle valve is modeled by the scheme in Fig. 1 [22]. We consider two tanks, of volumes $V_{\mathrm{i}}$ and $V_{\mathrm{o}}$, which represent the plenums before and after the compressor. The inlet tank stores uncompressed gas flowing from an upstream source through the inlet valve. The outlet tank receives gas from the compressor, which is then released to a downstream sink through the outlet valve. The inlet and outlet valves model the pressure drops due to the upstream process and the downstream sink. These are typically distributed pressure losses in long pipes or devices that require gas at pressure. The compressor is connected to the inlet tank, and the compressed gas flows via a duct, of length $L$ and section area $A$, before entering the outlet tank. We assume that there is no intermediate duct between the inlet of the compressor and the inlet tank. A recycle valve, with a 
controllable characteristic, operates from the outlet tank to the inlet tank to provide surge protection when necessary.

Since the simulation results are related to a real air compression system, in this paper, we will consider the particular gas to be air and the atmospheric conditions for upstream and downstream air. We remark that the validity of our results does not depend on the gas considered, but only on the availability of the mathematical model of Section II-A describing its physical properties.

\section{A. Mathematical Model}

The inlet tank pressure, $p_{\mathrm{i}}$, the outlet tank pressure, $p_{\mathrm{o}}$, and the mass flow rate through the compressor, $m$, are related by the model in (1) to the tank volumes and the sonic velocity at the inlet and outlet tank conditions, $a_{\mathrm{i}}$ and $a_{\mathrm{o}}$, typically assumed to be the sonic velocity at ambient conditions, $a_{\mathrm{s}}$. The compressor speed, $\omega$, is related to the compressor torque, $\tau_{\mathrm{c}}$, by the classic mechanical shaft equation, where the drive torque, $\tau_{\mathrm{d}}$, is assumed to be the manipulated input, $v$ is a friction coefficient, and $J$ is the mechanical inertia. A firstorder equation models the actuation of the recycle valve with a time constant $T_{\mathrm{r}}$, filtering the steady-state recycle mass flow $m_{\mathrm{r}, \mathrm{ss}}$ with the state variable $m_{\mathrm{r}}$. The mass flow rates through the inlet valve $m_{\mathrm{i}}$, the outlet valve $m_{\mathrm{o}}$, and the recycle valve in steady-state $m_{\mathrm{r}, \mathrm{ss}}$ are specified in (2). The compressor characteristics $\pi(\omega, m)$ and the torque profile $\tau_{\mathrm{c}}(\omega, m)$ are generally experimentally measured and approximated with polynomial equations [37]. Invariance to the inlet conditions can be achieved by writing the map in an appropriate coordinate system [38], [19].

The nonlinear system dynamics are given by

$$
\begin{aligned}
\dot{p}_{\mathrm{i}} & =\frac{a_{\mathrm{i}}^{2}}{V_{\mathrm{i}}}\left(m_{\mathrm{i}}\left(p_{\mathrm{i}}\right)-m+m_{\mathrm{r}}\right) \\
\dot{p}_{\mathrm{o}} & =\frac{a_{\mathrm{o}}^{2}}{V_{\mathrm{o}}}\left(-m_{\mathrm{o}}\left(p_{\mathrm{o}}\right)+m-m_{\mathrm{r}}\right) \\
\dot{m} & =\frac{A}{L}\left(\pi(\omega, m) p_{\mathrm{i}}-p_{\mathrm{o}}\right) \\
\dot{\omega} & =\frac{1}{J}\left(\tau_{\mathrm{d}}-\tau_{\mathrm{c}}(\omega, m)-v \omega\right) \\
\dot{m}_{\mathrm{r}} & =\frac{1}{T_{\mathrm{r}}}\left(m_{\mathrm{r}, \mathrm{ss}}\left(p_{\mathrm{i}}, p_{\mathrm{o}}, k_{\mathrm{r}}\right)-m_{\mathrm{r}}\right),
\end{aligned}
$$

where

$$
\begin{aligned}
m_{\mathrm{i}}\left(p_{\mathrm{i}}\right) & :=k_{\mathrm{i}} \sqrt{p_{\mathrm{atm}}-p_{\mathrm{i}}} \\
m_{\mathrm{o}}\left(p_{\mathrm{o}}\right) & :=k_{\mathrm{o}} \sqrt{p_{\mathrm{o}}-p_{\mathrm{atm}}} \\
m_{\mathrm{r}, \mathrm{ss}}\left(p_{\mathrm{i}}, p_{\mathrm{o}}, k_{\mathrm{r}}\right) & :=k_{\mathrm{r}} \sqrt{p_{\mathrm{o}}-p_{\mathrm{i}}} .
\end{aligned}
$$

The valve constants $k_{\mathrm{i}}, k_{\mathrm{o}}$, and $k_{\mathrm{r}}$ are determined by the valve throttle positions; hence, they are positive for open valves and zero when closed. The recycle valve constant, $k_{\mathrm{r}}$, is considered to be a manipulated input for surge control, while $k_{\mathrm{i}}$ and $k_{\mathrm{o}}$ are set by other system controllers to manage overall flow rates. The real actuator of the system is the valve throttle opening that will affect the valve constant, $k_{\mathrm{r}}$, with known characteristics. Nonreturn valves not displayed in the picture ensure that the mass flow rates $m_{\mathrm{i}}, m_{\mathrm{o}}$, and $m_{\mathrm{r}}$ cannot reverse the direction.

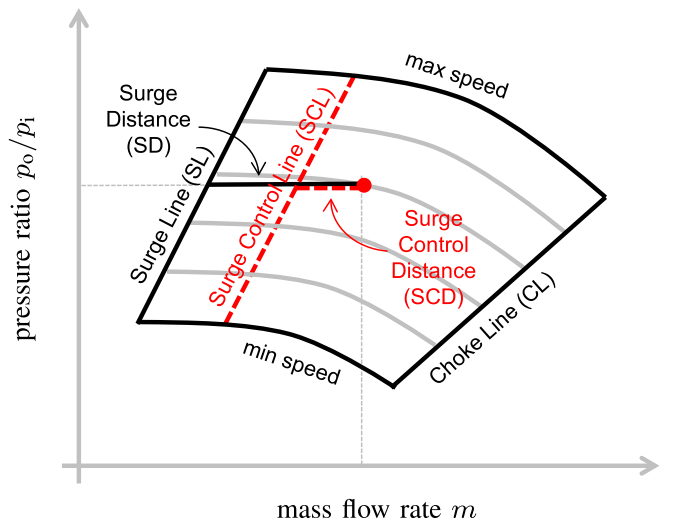

Fig. 2. Illustration of the most relevant lines in compressor maps.

Historically, compression systems have been extensively analyzed via nondimensional models [4], [8]. This is particularly convenient when the assumption of constant speed $\omega$ is made. In our particular case, however, we assume that the speed is a state of the system, variable and influenced by the action of the actuators. Hence, we prefer the control-oriented state space representation to the classical nondimensional formulation. Similar considerations can be found in the recent literature [10], [12], [21]-[23], [26].

In the following, we deal with constrained control techniques in the time domain, thus we consider a discretetime model for (1). Specifically, we generate an Euler discretization with zero-order hold assumption and sampling time $T_{\mathrm{s}}=50 \mathrm{~ms}$. The corresponding dynamical system is input affine, and the model can be written in a compact form as

$$
x^{+}=f(x)+g(x) u
$$

where the states $x$ and $u$ are expressed as the deviation from a desired operating condition

$$
\begin{aligned}
\dot{p}_{\mathrm{i}} & =\frac{a_{\mathrm{i}}^{2}}{V_{\mathrm{i}}}\left(m_{\mathrm{i}}\left(p_{\mathrm{i}}\right)-m+m_{\mathrm{r}}\right) \\
\dot{p}_{\mathrm{o}} & =\frac{a_{\mathrm{o}}^{2}}{V_{\mathrm{o}}}\left(-m_{\mathrm{o}}\left(p_{\mathrm{o}}\right)+m-m_{\mathrm{r}}\right) \\
\dot{m} & =\frac{A}{L}\left(\pi(\omega, m) p_{\mathrm{i}}-p_{\mathrm{o}}\right) \\
\dot{\omega} & =\frac{1}{J}\left(\tau_{\mathrm{d}}-\tau_{\mathrm{c}}(\omega, m)-v \omega\right) \\
\dot{m}_{\mathrm{r}} & =\frac{1}{T_{\mathrm{r}}}\left(m_{\mathrm{r}, \mathrm{ss}}\left(p_{\mathrm{i}}, p_{\mathrm{o}}, k_{\mathrm{r}}\right)-m_{\mathrm{r}}\right),
\end{aligned}
$$

$$
\begin{aligned}
x & :=\left[p_{\mathrm{i}}-\bar{p}_{\mathrm{i}}, p_{\mathrm{o}}-\bar{p}_{\mathrm{o}}, m-\bar{m}, \omega-\bar{\omega}, m_{\mathrm{r}}-\bar{m}_{\mathrm{r}}\right]^{\top}, \\
u & :=\left[\tau_{\mathrm{d}}-\bar{\tau}_{\mathrm{d}}, k_{\mathrm{r}}-\bar{k}_{\mathrm{r}}\right]^{\top},
\end{aligned}
$$

where the bar superscripts indicate desired steady-state values computed according to procedure in Section IIB. The inputs are subject to box constraints, due to the maximum/minimum providable drive torque on the rotor shaft and the maximum/null recycle valve characteristics, corresponding to fully open/closed throttle.

In Fig. 2, a typical compressor map is given. The pressure ratio $p_{\mathrm{o}} / p_{\mathrm{i}}$ is displayed as a function of the mass flow rate $m$. The SL is a physical limit that must not be exceeded, and it is generally experimentally determined. The SL is typically shifted by an experimentally determined safety margin to obtain the surge control line (SCL), shown as a dashed line. 
The distance of the operating point, displayed as a red dot, from the SL and the SCL defines, respectively, the SD and surge control distance (SCD) [18], [22], [39]. The choke line $(\mathrm{CL})$ is a maximum mass flow rate limit that, in contrast to the surge related lines, cannot be physically reached by the machine, and hence is not considered in the control problem. Finally, the gray curves represent speed isolines in steady-state conditions and are only for visualization purposes. Theoretically, the SL connects all the maxima of the speed lines. In industrial practice, though, the SL is determined experimentally by pushing the system toward surge at different speeds and recording the SL points before experiencing significant oscillations. Usually, simple straight lines are considered by the interpolation of the experimentally determined points. The controlled output of the system, $y$, is defined following typical industrial practice as the pressure ratio and the SCD. By assuming that the SCL has equation $p_{\mathrm{o}} / p_{\mathrm{i}}=\beta_{1} m+\beta_{2}$, with $\beta_{1}$ and $\beta_{2}$ determined experimentally, we have that

$$
y=h(x):=\left[\begin{array}{c}
p_{\mathrm{o}} / p_{\mathrm{i}} \\
m-\frac{1}{\beta_{1}}\left(\frac{p_{\mathrm{o}}}{p_{\mathrm{i}}}-\beta_{2}\right)
\end{array}\right] .
$$

Since the function $h$ is nonlinear, considering (mildly) nonlinear expressions for the SL does not significantly affect the nonlinearity of the problem.

\section{B. Steady-State Conditions}

The steady-state conditions for the system are easily obtained by imposing zero state derivatives in (1).

In the following, we derive the steady-state conditions corresponding to a desired output $\bar{y}$. According to (5), the first component gives the pressure ratio. Combining the first and second equation in (1) we have

$$
m_{\mathrm{i}}\left(\bar{p}_{\mathrm{i}}\right)=m_{\mathrm{o}}\left(\bar{p}_{\mathrm{o}}\right),
$$

which, combined with the first equation in (5), allows us to determine the steady-state inlet tank pressure as follows:

$$
\begin{aligned}
& \bar{p}_{\mathrm{i}}=P_{\mathrm{atm}} \frac{k_{\mathrm{i}}^{2}+k_{\mathrm{o}}^{2}}{k_{\mathrm{i}}^{2}+k_{\mathrm{o}}^{2} \bar{y}_{1}}, \\
& \bar{p}_{\mathrm{o}}=\bar{y}_{1} \bar{p}_{\mathrm{i}} .
\end{aligned}
$$

Then, the steady-state mass flow rate $\bar{m}$ is readily obtained from (5) as

$$
\bar{m}=\frac{1}{\beta_{1}}\left(\bar{y}_{1}-\beta_{2}\right)+\bar{y}_{2}
$$

while the steady-state recycle mass flow rate $\bar{m}_{\mathrm{r}}$ and recycle valve characteristic $\bar{k}_{\mathrm{r}}$ satisfy the following:

$$
\bar{m}_{\mathrm{r}}=\bar{m}-m_{\mathrm{i}}\left(\bar{p}_{\mathrm{i}}\right)=\bar{k}_{\mathrm{r}} \sqrt{\bar{p}_{\mathrm{o}}-\bar{p}_{\mathrm{i}}} .
$$

The steady-state compressor speed $\bar{\omega}$ comes from the solution of the third equation of (1). The compressor characteristics $\pi(\bar{\omega}, \bar{m})$ with fixed $\bar{m}$ is a second-order equation in $\bar{\omega}$, and it is solved analytically. For the structure of the equation, the two zeros are both real and only one is positive. Finally, the steady-state torque input, $\bar{\tau}_{\mathrm{d}}$, is readily obtained from the fourth equation in (1).

\section{Standard Industrial Control}

The state-of-the-art solution consists of independent process and antisurge controllers realized using PI or PID controllers. The process controller maintains a given set point by manipulating the drive torque or speed, whereas the antisurge controller is responsible for keeping the operation in the stable region of the compressor map by manipulating one or several recycle valves. The process controller typically has several modes of operation, e.g., discharge pressure control, discharge flow control, or pressure ratio control. Antisurge control is realized by computing the SCD shown in Fig. 2. The SCD is used as an error to drive the operating point to the SCL. The sign is adjusted such that operations on the right (safe) side of the SCL would correspond to negative valve characteristics $k_{\mathrm{r}}$. This is then clipped to $k_{\mathrm{r}}=0$ due to the input constraints. Desired operating points located on the left side of the SCL are reached by adding a positive offset to the recycle valve input.

Typical execution rates for process controllers are $0.5-1 \mathrm{~s}$, while antisurge control is much faster, with sampling time in the range of 50-200 ms depending on the system dynamics. In the present case study, the process controller is in pressure ratio control mode with a sampling time of $0.5 \mathrm{~s}$, whereas the antisurge controller is executed with a sampling time of $50 \mathrm{~ms}$. The conventional controller consists of an anti-windup PI scheme as follows:

$$
\begin{aligned}
& e_{k}=\bar{y}-y_{k} \\
& e_{\mathrm{int}, k}=e_{\mathrm{int}, k-1}+T_{\mathrm{s}} e_{k}-k_{\mathrm{awu}}\left(u_{k-1}-v_{k-1}\right) \\
& v_{k}=k_{\mathrm{p}} e_{k}+k_{\mathrm{i}} e_{\mathrm{int}, k} \\
& u_{k}=\operatorname{sat}\left(v_{k}, u_{\min }, u_{\text {max }}\right)
\end{aligned}
$$

where the sat $(\cdot)$ operator projects $v_{k}$ onto $\left[u_{\min }, u_{\max }\right]$.

To improve the system responses, a simple gain scheduling logic was introduced for both controllers. For the antisurge controller, the gains are multiplied by a factor of 1.5 if the absolute error is smaller than $25 \%$, whereas for the process controller, the same factor is used in an error band of $\pm 5 \%$.

\section{Review of Contractive Nonlinear Model Predictive Control}

MPC has been shown to be an effective approach for the regulation of compression systems [21]-[23], [40], where quadratic cost functions are typically used to tradeoff between performance and actuator usage.

Maintaining the process within safe limits is a critical requirement, for instance to avoid surge phenomena. In this section, we review and apply the contractive MPC approach [27] to ensure closed-loop stability. With this aim, we formulate the decrease (contraction) of a quadratic CLF as an additional constraint. Then, in Section III-B, we introduce a numerical procedure for assessing recursive feasibility of such a constraint.

\section{A. Formulation of the Control Problem}

Recursive feasibility and in turn asymptotic stability can be guaranteed by imposing a decrease (contraction) of a factor $\alpha \in[0,1)$ of a suitable CLF [27] every $N$ discrete-time steps. 
Thus, the contraction constraint is time-varying, as it appears in the nonlinear MPC problem at time steps that are multiples of $N$.

By denoting the current time step as $t_{k}$, we define $t_{\tilde{k} \mid k}$ as the predicted time step in which the contractive constraint is enforced. For example, at the first time step $t_{0}$, the contractive constraint is $N$ samples in the future, hence $\tilde{k}=N$. In the following time step, $t_{1}$, we have $\tilde{k}=N-1$, and then recursively up to the time step $t_{N}$, where we have $\tilde{k}=2 N$. Hence, given the current state $x_{k}$, the contractive MPC problem can be written as follows [27]:

$$
\begin{array}{cl}
\min _{\left\{u_{k+j \mid k}\right\}_{j=0}^{N-1}} & \sum_{j=0}^{N-1}\left\{\left\|x_{k+j \mid k}\right\|_{Q}^{2}+\left\|u_{k+j \mid k}\right\|_{R}^{2}\right\}+\left\|x_{k+N \mid k}\right\|_{P}^{2} \\
\text { s.t. } \quad & x_{k+j+1 \mid k}=f\left(x_{k+j \mid k}\right)+g\left(x_{k+j \mid k}\right) u_{k+j \mid k} \\
& u_{\min } \leq u_{k+j \mid k} \leq u_{\max } \quad \forall j \in\{0, \ldots, N-1\} \\
& \left\|x_{\tilde{k} \mid k}\right\|_{P}^{2} \leq \alpha\left\|x_{\tilde{k}-N}\right\|_{P}^{2},
\end{array}
$$

where $\alpha \in[0,1)$ is the contraction parameter, $x_{k+j \mid k}$ and $u_{k+j \mid k}$, respectively, denote the state and the input predicted $j$ time steps ahead, and the state $x_{\tilde{k} \mid k}$ denotes the predicted state at the time $t_{\tilde{k} \mid k}$.

When the first component $u_{k \mid k}$ of the optimal solution to (7) is applied to the system, asymptotic stability and recursive feasibility are guaranteed if the function $V(\cdot):=\|\cdot\|_{P}^{2}$ is a CLF. That is, for each initial state, there exists a feasible control sequence that contracts $V$ after $N$ time steps [27, Th. 1].

\section{B. Numerical Test of Recursive Feasibility}

In our numerical simulations, the problem of finding $P \succ 0$ and tuning $\alpha \in[0,1)$ is solved numerically. Let us start from the knowledge of a CLF $V(x):=x^{\top} P x=\|x\|_{P}^{2}$ for the system linearized around the origin, i.e., the desired equilibrium. For instance, the matrix $P$ can be the solution of the Riccati equation, with weight matrices $Q$ and $R$.

To estimate the region in which $V$ is a CLF for the nonlinear system, we grid the $n$-dimensional state space around the origin. Given a desired contraction factor $\alpha \in[0,1)$, we check the Lyapunov-decrease condition for every grid point $x$, that is

$$
V(f(x)+g(x) u) \leq \alpha V(x)
$$

for some $u \in\left[u_{\min }, u_{\max }\right] \subset \mathbb{R}^{m}$, which is equivalent to

$$
(f(x)+g(x) u)^{\top} P(f(x)+g(x) u) \leq \alpha x^{\top} P x .
$$

Now, for a given state $x \in \mathbb{R}^{n}$, the existence of a feasible control input $u \in\left[u_{\min }, u_{\max }\right] \subset \mathbb{R}^{m}$ such that (9) holds can be determined by solving the QP

$$
\begin{gathered}
u^{*}(x):=\arg \min _{u \in \mathbb{R}^{m}} u^{\top} g(x)^{\top} P g(x) u+2 f(x)^{\top} P g(x) u \\
\text { s.t. } u_{\min } \leq u \leq u_{\max }
\end{gathered}
$$

and then checking the inequality

$$
J^{*}(x):=J\left(u^{*}(x)\right) \leq \alpha x^{\top} P x-f(x)^{\top} P f(x) .
$$

The numerical approach that we propose is to check the inequality in (11) over a finite number of state vectors on a sufficiently fine grid. Specifically, we take $x \in \mathbb{R}^{n}$ as a generic grid vector and $u^{\star}=u^{\star}(x)$. Then, we consider the Lyapunovdecrease condition in an $\varepsilon$-neighborhood of $x$, that is

$$
\max _{\delta \in \varepsilon \mathbb{B}} V\left(f(x+\delta)+g(x+\delta) u^{\star}\right)-\alpha V(x+\delta) \leq 0 .
$$

By defining $L(x)$ as the local Lipschitz constant of the function $V\left(f(\cdot)+g(\cdot) u^{\star}\right)-\alpha V(\cdot)$ relative to the neighborhood $x+\varepsilon \mathbb{B}$, (12) can be obtained as follows:

$$
\begin{aligned}
& \max _{\delta \in \varepsilon \mathbb{B}} V\left(f(x+\delta)+g(x+\delta) u^{\star}\right)-\alpha V(x+\delta) \\
& =\max _{\delta \in \varepsilon \mathbb{B}} V\left(f(x)+g(x) u^{\star}\right)-\alpha V(x) \\
& +V\left(f(x+\delta)+g(x+\delta) u^{\star}\right)-\alpha V(x+\delta) \\
& -\left(V\left(f(x)+g(x) u^{\star}\right)-\alpha V(x)\right) \\
& \leq V\left(f(x)+g(x) u^{\star}\right)-\alpha V(x) \\
& +\varepsilon \max _{\delta \in \varepsilon \mathbb{B}} \frac{1}{\varepsilon} \mid V\left(f(x+\delta)+g(x+\delta) u^{\star}\right)-\alpha V(x+\delta) \\
& -\left(V\left(f(x)+g(x) u^{\star}\right)-\alpha V(x)\right) \\
& =V\left(f(x)+g(x) u^{\star}\right)-\alpha V(x)+\varepsilon L(x) \leq 0 .
\end{aligned}
$$

Note that as usual, the last inequality in (13) does not hold in a neighborhood of the origin. In fact, the desired steadystate point $x=0$ is such that $f(0)=0$, hence $u^{\star}=0$ from (10). As $V(0)=0$, (13) becomes $\varepsilon L(0) \leq 0$, with $L(0)=\max _{\delta \in \varepsilon \mathbb{B}}(1 / \varepsilon)|V(f(\delta))-\alpha V(\delta)|>0$. Therefore, by computing an upper bound to $L(x)$, we derive the Lyapunov-decrease condition in (13) to be checked outside a neighborhood of the origin.

We now propose a procedure to numerically determine the set of states that if reachable in at most $N$ steps provides a certificate of asymptotic stability and recursive feasibility for the MPC algorithm.

Given the CLF $V(x)$ and the level set value $\alpha_{1}$, we define the region

$$
\mathcal{E}_{1}=\left\{x \in \mathbb{R}^{5} \mid V(x) \leq \alpha_{1}\right\}
$$

The numerical test aims at deriving the largest region $\mathcal{E}_{1}$ in which the constraint in (8) is satisfied for any point $x \in \mathcal{E}_{1}$, for some feasible input $u(x) \in\left[u_{\min }, u_{\max }\right]$.

Since the condition in (12) cannot be satisfied for $x=0$, we denote the small neighborhood of the origin in which the condition does not hold as

$$
\mathcal{E}_{2}=\left\{x \in \mathbb{R}^{5} \mid V(x) \leq \alpha_{2}\right\}
$$

for some constant $\alpha_{2}>0$.

We determine the numerical values $\alpha_{1}$ and $\alpha_{2}$ through Algorithm 1. The algorithm tests all points $\left\{x_{i}\right\}_{i}$ in a grid $\mathcal{X}$ and returns the smallest level set value $\bar{\alpha}:=V\left(x_{i}\right)$ such that $x_{i}$ is unfeasible, i.e., not satisfying the Lyapunov constraint (11). Therefore, the algorithm ensures that there exists no point in the region $\overline{\mathcal{E}}=\left\{x \in \mathbb{R}^{5} \mid V(x)<\bar{\alpha}\right\}$ that does not satisfy (11). In addition, the algorithm returns the CLF values $\left(V_{k}\right)_{k}$ and flags $\left(r_{k}\right)_{k}$ that are used to compute $\alpha_{1}$ and $\alpha_{2}$ as follows:

$$
\begin{aligned}
\alpha_{2}:=\min _{k} & V_{k} \\
\text { s.t. } & r_{k}=2 ;
\end{aligned}
$$




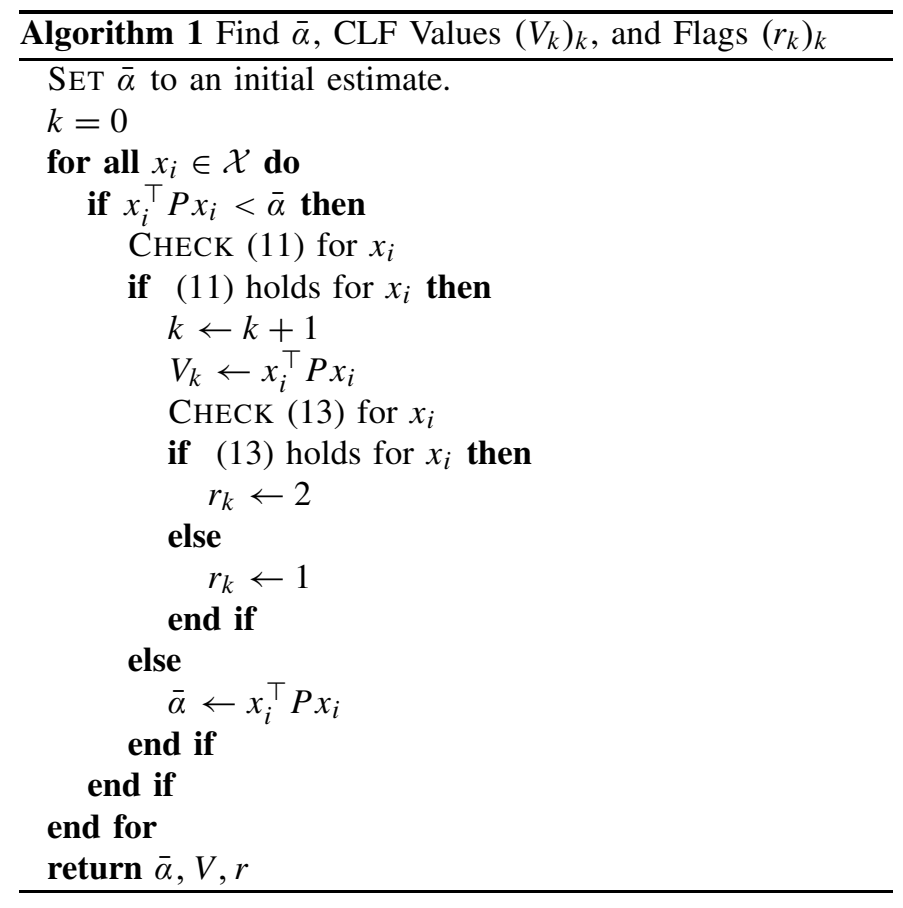

$$
\begin{aligned}
\alpha_{1}:=\max _{k} & V_{k} \\
\text { s.t. } & V_{k}<\bar{\alpha} \\
& r_{k}=2 \quad \forall k \in\left\{l \mid \alpha_{2} \leq V_{l} \leq V_{k}\right\} .
\end{aligned}
$$

The optimization problems in (16) and (17) can be solved efficiently, e.g., by sorting and inspecting the values $\left(V_{k}\right)_{k}$ and $\left(r_{k}\right)_{k}$. Note that the desired reference point affects the resulting level sets $\alpha_{1}, \alpha_{2}$, and $\bar{\alpha}$. In fact, choosing a steadystate null recycle valve actuation, $\bar{k}_{\mathrm{r}}=0$, corresponding to a null recycle mass flow rate, $\bar{m}_{\mathrm{r}}=0$, shrinks the level set $\alpha_{1}$ to zero, because the system is operated on its boundary.

\section{Numerical Example}

In this section, we consider the equilibrium with specifications $\bar{p}_{\mathrm{o}}=1.2$ bar and recycle valve opening $\bar{k}_{\mathrm{r}}=0.2 k_{\mathrm{r} \text { max }}$. Algorithm 1 is applied to determine $\bar{\alpha}=7.714 \mathrm{e}-4$. We sort in increasing order the Lyapunov function evalutions and the corresponding indexes derived by Algorithm 1, and obtain $\alpha_{2}:=7.368 \mathrm{e}-5$ and $\alpha_{1}:=2.322 \mathrm{e}-4$.

Next, we consider the requirement that the state can reach the ellipsoid $\mathcal{E}_{1}(14)$ in $N$ prediction steps, that is

$$
\begin{aligned}
& \min _{\left\{u_{k+j \mid k}\right\}_{j=0}^{N-1}} \sum_{j=0}^{N-1}\left\{\left\|x_{k+j \mid k}\right\|_{Q}^{2}+\left\|u_{k+j \mid k}\right\|_{R}^{2}\right\}+\left\|x_{k+N \mid k}\right\|_{P}^{2} \\
& \text { s.t. } \quad x_{k+j+1 \mid k}=f\left(x_{k+j \mid k}\right)+g\left(x_{k+j \mid k}\right) u_{k+j \mid k} \\
& u_{\text {min }} \leq u_{k+j \mid k} \leq u_{\max } \forall j \in\{0, \ldots, N-1\} \\
& \left\|x_{k+N \mid k}\right\|_{P} \leq \alpha_{1} \text {. }
\end{aligned}
$$

The feasibility of (18), together with the feasibility of (7), ensures recursive feasibility of the MPC for every successive time step.

Fig. 3 shows a state trajectory on the compressor map under the contractive MPC. The simulation is based on the realistic test bench described in more detail in the Appendix.

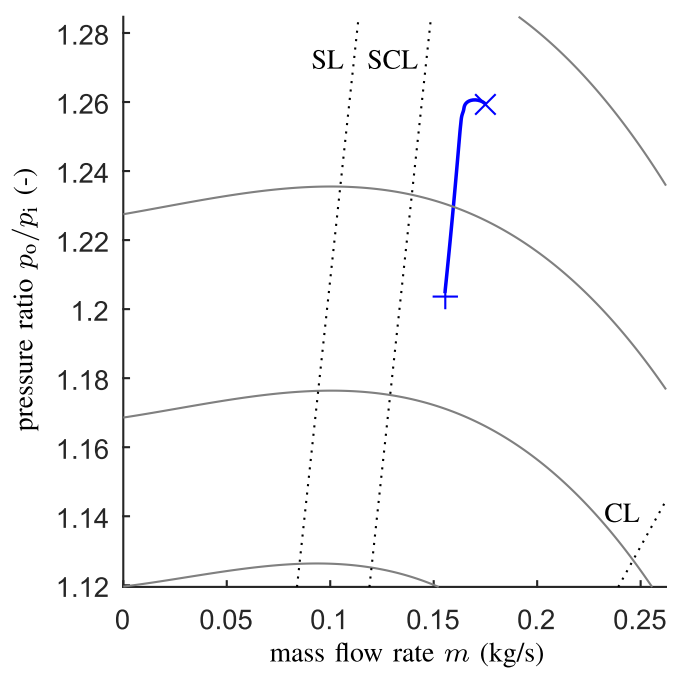

Fig. 3. Compressor map: the contractive nonlinear MPC ensures asymptotic stability and recursive feasibility: the closed-loop trajectory converges to the desired equilibrium $(+)$ starting from an initial condition $(x)$ which is feasible for both (18) and (7).

The prediction horizon is $N=20$ steps, with sampling time $T_{\mathrm{s}}=50 \mathrm{~ms}$, and the simulation time is $t=50 \mathrm{~s}$. We solve the program in (7) via the first-order algorithm in [41] that specifically exploits the structure of the problem. The system modeling, including the SL, SCL, and CL definitions, follows from [23].

An estimate of the level of conservativeness introduced by the approach can be obtained by checking the contractive inequality in (8) after computing the optimal solution of (7). For this numerical example, we observe that the contractive constraint never becomes active, and in particular, the distance from the boundary of the constraint shrinks as the operating point reaches the desired target. The implications are twofold: first, the MPC considered in this particular simulation would also be closed-loop stable without the addition of the contractive constraint. Moreover, the conservativeness introduced allows for possible asymptotically decaying disturbances in the dynamics [27].

\section{REVIEW OF OFFSET-FreE MODEL PREDICTIVE CONTROL}

While the contractive MPC formulation requires perfect knowledge of the system dynamics and at most asymptotically vanishing disturbances, in real industrial compression plants, more severe conditions are experienced, e.g., due to external unmodeled disturbances. Examples include downstream pressure drops, or model mismatch.

In this section, we discuss the offset-free MPC formulation to ensure that the closed-loop dynamics converge to the desired operating point, even in the presence of model mismatch and nonasymptotically decaying disturbance inputs. We consider two approaches to handle the resulting computational complexity: the linearized MPC approach (described in Section IV-A) and the SQP approach [29]. We assume that a sufficiently long prediction horizon is used for ensuring closed-loop stability. 


\section{A. Linearized Model Predictive Control}

One MPC approach relies on the linearization of the nonlinear system dynamics in (3) around the target steady-state point $(\bar{x}, \bar{u})$, computed from the desired output $\bar{y}$ according to the procedure in Section II-B. The linear time-invariant model can be rewritten as

$$
\begin{aligned}
\Delta x^{+} & =\bar{A} \Delta x+\bar{B} \Delta u \\
\Delta y & =\bar{C} \Delta x
\end{aligned}
$$

where $\Delta x:=x-\bar{x}, \Delta u:=u-\bar{u}$, and $\Delta y:=y-\bar{C} \bar{x}$, and $\bar{A}:=(\partial f / \partial x)(\bar{x})+(\partial g / \partial x)(\bar{x}) \bar{u}, \bar{B}:=g(\bar{x}), \bar{C}:=$ $(\partial h / \partial x)(\bar{x})$.

In the presence of persistent disturbances or model mismatch, such an MPC approach results in an asymptotic nonzero offset. To avoid this, offset-free MPC approaches [34], [35] consider an augmented model with trivial disturbance dynamics, where an estimate of the augmented state can be obtained via a filter (e.g., Kalman filter). In our case study, we augment the linearized model as

$$
\begin{aligned}
{\left[\begin{array}{c}
\Delta x \\
d
\end{array}\right]^{+} } & =\left[\begin{array}{cc}
\bar{A} & B_{\mathrm{d}} \\
0 & I
\end{array}\right]\left[\begin{array}{c}
\Delta x \\
d
\end{array}\right]+\left[\begin{array}{c}
\bar{B} \\
0
\end{array}\right] \Delta u \\
\Delta y & =\left[\begin{array}{ll}
\bar{C} & C_{\mathrm{d}}
\end{array}\right]\left[\begin{array}{c}
\Delta x \\
d
\end{array}\right]
\end{aligned}
$$

where $d \in \mathbb{R}^{2}$, thus $B_{\mathrm{d}} \in \mathbb{R}^{5 \times 2}$ and $C_{\mathrm{d}} \in \mathbb{R}^{2 \times 2}$. From our numerical experience, a good choice for the design of the disturbance model is a pure input disturbance, that is $B_{\mathrm{d}}=B$ and $C_{\mathrm{d}}=0$, since with this choice, the disturbance is filtered by the dynamics. We have experienced poorer performance with a pure output disturbance model, i.e., $B_{\mathrm{d}}=0$ and $C_{\mathrm{d}}=I$, because of the presence of poles of the linearized model characteristic polynomial close to $(1,0)$ [36].

For the design of the steady-state Kalman filter, we follow the standard procedure: an additive zero-mean process and measurement white-noise are considered in the equations, then the discrete algebraic Riccati equation is solved to obtain the update gain $L=\left[L_{\mathrm{x}} ; L_{\mathrm{d}}\right]$. This design yields a full rank square matrix $L_{\mathrm{d}}$ that automatically satisfies one of the offset-free tracking conditions [34]. Since in our application the state is easily measured, we assume the measurement noise covariance to be significantly smaller than the process covariance.

Given the current estimate of the disturbance $\hat{d}_{k \mid k}$, the following model inversion gives the updated state and input set points $\bar{x}_{k \mid k}$ and $\bar{u}_{k \mid k}$ at time $k$ :

$$
\left[\begin{array}{cc}
I-\bar{A}-\bar{B} \\
\bar{C} & 0
\end{array}\right]\left[\begin{array}{c}
\bar{x}_{k \mid k} \\
\bar{u}_{k \mid k}
\end{array}\right]=\left[\begin{array}{c}
B_{\mathrm{d}} \hat{d}_{k \mid k} \\
-C_{\mathrm{d}} \hat{d}_{k \mid k}+\Delta \bar{y}
\end{array}\right],
$$

where due to the expression of our function $h$, the matrix on the left-hand side is square and invertible, and the reference $\Delta \bar{y}:=\bar{y}-\bar{C} \bar{x}$.

Note that the resulting input $\bar{u}_{k \mid k}$ could be unfeasible for the actuator limit. In this case, the control would not be offsetfree and only a point close to the desired output $\bar{y}$ would be reached. Moreover, the existing input constraint must not be active at the steady-state point in order to obtain offset-free tracking [34]. In industrial practice, it is often the case that the desired operating point corresponds to null recycle valve actuation, thus resulting in one active input constraint. From our simulation experience, the actual offset is negligibly small and completely satisfactory for practical purposes.

The linearized MPC problem is then expressed as

$$
\begin{array}{cc}
\min _{\left\{\Delta u_{k+j \mid k}\right\}_{j=0}^{N-1}} & \sum_{j=0}^{N-1}\left\{\left\|\Delta y_{k+j \mid k}-\bar{y}\right\|_{Q_{\mathrm{y}}}^{2}+\left\|\Delta u_{k+j \mid k}-\bar{u}_{k \mid k}\right\|_{R}^{2}\right\} \\
& +\left\|\Delta y_{k+N \mid k}-\bar{y}\right\|_{Q_{\mathrm{y}}}^{2} \\
\text { s.t. } \quad \Delta x_{k \mid k}=\Delta \hat{x}_{k \mid k} & \bar{A}\left(\Delta x_{k+j \mid k}-\bar{x}_{k \mid k}\right) \\
& \quad+\bar{B}\left(\Delta x_{k+j+1 \mid k}-\bar{x}_{k \mid k}=\bar{u}_{k \mid k}\right) \\
& u_{\min } \leq \Delta u_{k+j \mid k}+\bar{u} \leq u_{\max } \\
& \forall j \in\{0, \ldots, N-1\} \\
\Delta y_{k+j \mid k}=\bar{C} \Delta x_{k+j \mid k} \quad \forall j \in\{0, \ldots, N\},
\end{array}
$$

where $Q_{y}, R \succ 0$. The cost function trades off the distance from the SL with the usage of the actuators, and in particular the emergency recycle valve.

\section{B. Nonlinear Model Predictive Control via Sequential Quadratic Programming}

The offset-free MPC approach also applies to the nonlinear case [36], via the internal model principle. Based on the current disturbance estimate and the desired reference, the target state and input steady-state conditions are computed. Finally, the nonlinear MPC tracks the computed target state and input, subject to the augmented dynamics and the original constraints. Analogously to the linear case, we consider a pure input disturbance model

$$
\begin{aligned}
x^{+} & =f(x)+g(x)(u+d) \\
d^{+} & =d \\
y & =h(x) .
\end{aligned}
$$

We consider an extended Kalman filter due to the nonlinear dynamics. Specifically, given the current estimate of the disturbance $\hat{d}_{k \mid k}$ and the desired output reference $\bar{y}$, the state and input set points at time $k,\left(\bar{x}_{k \mid k}, \bar{u}_{k \mid k}\right)$, are given by

$$
\begin{aligned}
\bar{x}_{k \mid k} & =f\left(\bar{x}_{k \mid k}\right)+g\left(\bar{x}_{k \mid k}\right)\left(\bar{u}_{k \mid k}+\hat{d}_{k \mid k}\right) \\
\bar{y} & =h\left(\bar{x}_{k \mid k}\right) .
\end{aligned}
$$

As in the linear case, the derived set point needs to satisfy the original constraint for offset-free control and uniqueness of the set point, which holds true in our application. The nonlinear MPC problem is then

$$
\begin{aligned}
\min _{\left\{u_{k+j \mid k}, s_{j}\right\}_{j=0}^{N-1}} & \sum_{j=0}^{N-1}\left\{\left\|y_{k+j \mid k}-\bar{y}\right\|_{Q_{\mathrm{y}}}^{2}+\left\|u_{k+j \mid k}-\bar{u}_{k \mid k}\right\|_{R}^{2}\right. \\
& \left.+\lambda s_{j}^{2}\right\}+\left\|y_{k+N \mid k}-\bar{y}\right\|_{Q_{\mathrm{y}}}^{2}+\lambda s_{N}^{2} \\
\text { s.t. } \quad & x_{k \mid k}=\hat{x}_{k \mid k} \quad \\
& x_{k+j+1 \mid k}=f\left(x_{k+j \mid k}\right) \\
& \quad+g\left(x_{k+j \mid k}\right)\left(u_{k+j \mid k}+\hat{d}_{k \mid k}\right) \\
& u_{\min } \leq u_{k+j \mid k} \leq u_{\max } \quad \forall j \in\{0, \ldots, N-1\} \\
& y_{k+j \mid k}=h\left(x_{k+j \mid k}\right) \quad \forall j \in\{0, \ldots, N\} \\
& y_{k+j \mid k}(2) \geq-d-s_{j}, \quad s_{j} \geq 0 \forall j \in\{0, \ldots, N\} .
\end{aligned}
$$


The last constraint takes the SL explicitly into account. Since the SCL is obtained by a shift $d>0$ of the SL, by constraining the SCD output $y_{k+j \mid k}(2)$ to be larger than $-d$, we impose that the system operates on the right of the SL. The slacks $s_{j}$ make the constraint soft via a penalty term $\lambda$ to avoid infeasibility.

To handle the problem of nonconvexity, we consider the SQP approach [29]. Starting from an initial guess $\boldsymbol{u}_{k}^{(i)}:=\left[u_{k \mid k}^{(i)} ; \ldots ; u_{k+N-1 \mid k}^{(i)}\right]$ for the control input sequence at time step $k$, and the corresponding state evolution $\boldsymbol{x}_{k}^{(i)}:=\left[x_{k+1 \mid k}^{(i)} ; \ldots ; x_{k+N \mid k}^{(i)}\right]$, we define the matrices $A_{k+j \mid k}^{(i)}$, $B_{k+j \mid k}^{(i)}$, and $C_{k+j \mid k}^{(i)}$, and vectors $f_{k+j \mid k}^{(i)}$ and $h_{k+j \mid k}^{(i)}$ from the time-varying linearization around the state-input trajectory $\left(\boldsymbol{x}_{k}^{(i)}, \boldsymbol{u}_{k}^{(i)}\right)$ and disturbance $\hat{d}_{k \mid k}$, that is

$$
\begin{aligned}
A_{k+j \mid k}^{(i)} & :=\frac{\partial f}{\partial x}\left(x_{k+j \mid k}^{(i)}\right)+\frac{\partial g}{\partial x}\left(x_{k+j \mid k}^{(i)}\right)\left(u_{k+j \mid k}^{(i)}+\hat{d}_{k \mid k}\right) \\
B_{k+j \mid k}^{(i)} & :=g\left(x_{k+j \mid k}^{(i)}\right) \\
f_{k+j \mid k}^{(i)} & :=f\left(x_{k+j \mid k}^{(i)}\right)+g\left(x_{k+j \mid k}^{(i)}\right)\left(u_{k+j \mid k}^{(i)}+\hat{d}_{k \mid k}\right)-x_{k+j+1 \mid k}^{(i)} \\
C_{k+j \mid k}^{(i)} & :=\frac{\partial h}{\partial x}\left(x_{k+j \mid k}^{(i)}\right), \quad h_{k+j \mid k}^{(i)}:=h\left(x_{k+j \mid k}^{(i)}\right)-y_{k+j \mid k}^{(i)} .
\end{aligned}
$$

Then, one has to update the solution by finding the deviations $\quad \boldsymbol{\Delta} \boldsymbol{u}_{k}^{(i)} \quad:=\left[\Delta u_{k \mid k}^{(i)} ; \ldots ; \Delta u_{k+N-1 \mid k}^{(i)}\right]$ and $\Delta s^{(i)}:=\left[\Delta s_{0}^{(i)}, \ldots, \Delta s_{N}^{(i)}\right]$ that solve the optimization problem

$$
\begin{aligned}
\min _{\Delta \boldsymbol{u}_{k}, \boldsymbol{\Delta} \boldsymbol{s}} & \sum_{j=0}^{N-1}\left\{\left(Q_{\mathrm{y}, j}^{(i)} \Delta y_{k+j \mid k}+2 Q_{\mathrm{y}}\left(y_{k+j \mid k}^{(i)}-\bar{y}\right)\right)^{\top} \Delta y_{k+j \mid k}\right. \\
& +\left(R_{j}^{(i)} \Delta u_{k+j \mid k}+2 R\left(u_{k+j \mid k}^{(i)}-\bar{u}_{k \mid k}\right)\right)^{\top} \Delta u_{k+j \mid k} \\
& \left.+\left(\lambda^{(i)} \Delta s_{j}+2 \lambda s_{j}^{(i)}\right) \Delta s_{j}\right\}+\left(\lambda^{(i)} \Delta s_{N}+2 \lambda s_{N}^{(i)}\right) \Delta s_{N} \\
& +\left(Q_{\mathrm{y}, N}^{(i)} \Delta y_{k+N \mid k}+2 Q_{\mathrm{y}}\left(y_{k+N \mid k}^{(i)}-\bar{y}\right)\right)^{\top} \Delta y_{k+N \mid k} \\
\text { s.t. } \quad & \Delta x_{k+j+1 \mid k}=A_{k+j \mid k}^{(i)} \Delta x_{k+j \mid k}+B_{k+j \mid k}^{(i)} \Delta u_{k+j \mid k} \quad+f_{k+j \mid k}^{(i)} \\
& \Delta u_{k+j \mid k}+u_{k+j \mid k}^{(i)} \in\left[u_{\min }, u_{\max }\right] \forall j \in\{0, \ldots, N-1\} \\
& \Delta y_{k+j \mid k}=C_{k+j \mid k}^{(i)} \Delta x_{k+j \mid k}+h_{k+j \mid k}^{(i)} \forall j \in\{0, \ldots, N\} \\
& \Delta y_{k+j \mid k}(2)+y_{k+j \mid k}^{(i)}(2) \geq-d-\Delta s_{j}-s_{j}^{(i)} \\
& \Delta s_{j}+s_{j}^{(i)} \geq 0 \quad \forall j \in\{0, \ldots, N\} \\
& \left\|\Sigma^{(i)} \quad \boldsymbol{\Delta} \boldsymbol{u}_{k}^{(i)}\right\|_{\infty} \leq \delta^{(i)},
\end{aligned}
$$

which, together with the resulting state and output deviations, $\boldsymbol{\Delta} \boldsymbol{x}_{k}^{(i)}$ and $\boldsymbol{\Delta} \boldsymbol{y}_{k}^{(i)}$, update the corresponding trajectories.

The last constraint in (26), $\left\|\Sigma^{(i)} \boldsymbol{\Delta} \boldsymbol{u}_{k}^{(i)}\right\|_{\infty} \leq \delta^{(i)}$, with $\Sigma^{(i)} \succ 0$ is added to the MPC problem to find a control input sequence $\boldsymbol{u}_{k}^{(i+1)}$, which is sufficiently close to $\boldsymbol{u}_{k}^{(i)}$, based on a trust-region method [28]. The weight matrices $\left(Q_{\mathrm{y}, j}^{(i)}, R^{(i)}\right)$ should be chosen as the corresponding Hessians of the Lagrangian function associated with the original optimal control problem. However, they can be approximated with $\left(Q_{\mathrm{y}}, R\right)$ to avoid their online computation [29].
The QP in (26) is solved iteratively $M$ times, where $M$ is a priori chosen and/or tuned empirically; once $M$ iterations are completed, the first component $u_{k+1 \mid k}^{(M)}$ of the control input sequence $\boldsymbol{u}_{k}^{(M)}$ is applied to the system. Then, the next initial input sequence $\boldsymbol{u}_{k+1}^{(0)}$ for (26) can be obtained by shifting the sequence $\boldsymbol{u}_{k}^{(M)}$ by one stage, and using the discrete-time Linear-Quadratic Regulator (LQR) solution for the calculation of the final input $u_{k+N \mid k+1}^{(0)}$. At the very first sampling time $(k=0)$, since no previous control input trajectory is available, one can construct $\boldsymbol{u}_{0}^{(0)}$ based on the projected LQR solution. For $M=1$, one recovers the real-time iteration approach [30], [31].

\section{Simulation Results}

The performance of the offset-free controllers presented in Section IV are compared in closed-loop simulations to the industrial control presented in Section II-C. In contrast to the SQP MPC, which uses explicit Euler integration, the nonlinear MPC employs Runge-Kutta (RK4) discretization for further comparison of the closed-loop effect of the integration. The conventional state-of-the-art controller is designed as follows: the antisurge control updates with a sampling time of $T_{\mathrm{s}}=50 \mathrm{~ms}$. The torque control, employed to reach the desired pressure ratio, updates with a sampling time of $T_{\mathrm{s}, \tau}=500 \mathrm{~ms}$. The torque and valve-coupled MPC controllers that we propose operate at a sampling time of $T_{\mathrm{s}}=50 \mathrm{~ms}$ and are designed to see with $N=20$ prediction steps ahead. The output and input cost matrices, $Q_{\mathrm{y}}$ and $R$, are designed such that the control aggressiveness is similar to the state-of-the-art control and in order to use mostly the torque input.

The simulations are performed starting from a steady-state point on the compressor map, corresponding to an outlet pressure of 1.3 bar, and moving to a desired different steadystate point, at the pressure of 1.15 bar, computed following a procedure analogous to the one in Section II-B.

Section V-A considers the nominal case, i.e., assuming exact knowledge of the model parameters in Section II. In Section $\mathrm{V}-\mathrm{B}$, typical disturbances and model mismatch are considered. Details about the required computational times are reported in Section V-C.

\section{A. Nominal Case}

As in the previous simulation of Section III-C, the identified compressor map for the simulation test bench is given in Fig. 4. Speed isolines computed from the compressor characteristics, $\pi(\omega, m)$, are displayed for visualization purposes, as well as the SL and the CL. The SCL is drawn at a safety distance from the SL by a shift of an experimentally determined offset.

As a first scenario, we consider a target point located in a safe zone on the right side of the SCL, thanks to a large opening of the inlet and outlet valves. From Fig. 4, we notice that the MPC-based controllers have a similar closed-loop response to the conventional controller. Moreover, setting $M=1$ is already enough for the SQP MPC in (26) to get a closed-loop response essentially equal to the nonlinear MPC in (24), solved with an interior-point method. Furthermore, we notice that in this set of simulations, the effects of a 


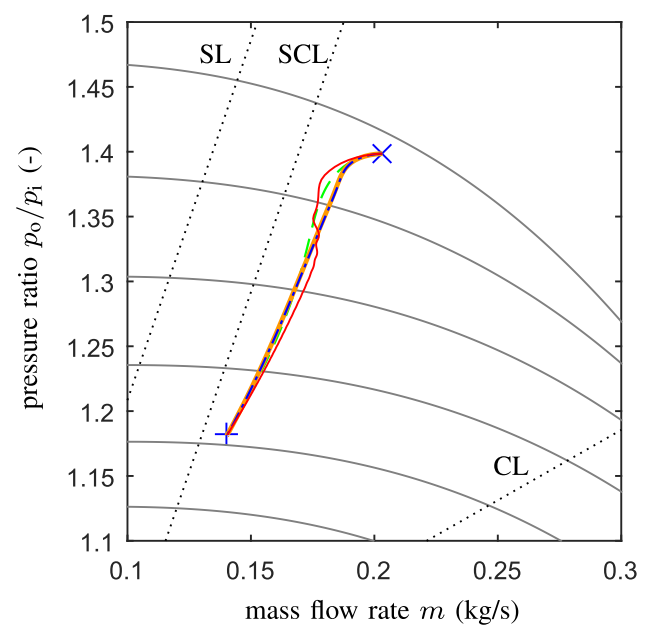

Fig. 4. Nominal case (first scenario) - Compressor map: the red line corresponds to the standard PID control, the dashed light green line corresponds to the linearized MPC, dash-dot blue to the nonlinear MPC and the orange thick to the SQP MPC.

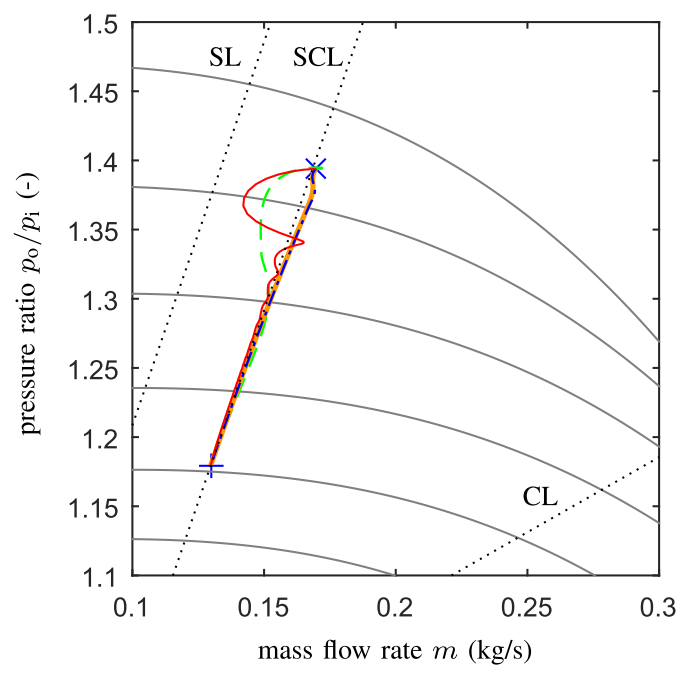

Fig. 5. Nominal case (second scenario) - Compressor map: the red line corresponds to the standard PID control, the dashed light green line corresponds to the linearized MPC, dash-dot blue to the nonlinear MPC and the orange thick to the SQP MPC.

different discretization are barely noticeable. The industrial antisurge control does not employ the recycle valve actuation at all, since the SCL is never crossed, and neither does the MPC, as a result of a cost matrix tuning that penalizes the usage of the emergency recycle valve.

A second scenario is displayed in Fig. 5, where the target equilibrium is located on the SCL, due to different external conditions, i.e., throttle opening of the input and output valves. This desired steady-state operating point requires a positive recycle valve actuation, according to Section II-B. This is due to smaller openings of the inlet and outlet valve compared with the previous case, to simulate a more extreme external process requirement. The corresponding closed-loop response is displayed in a compressor map in Fig. 5 and the torque and recycle valve actuation in Fig. 6.

In this case, the conventional control and the linearized MPC move closer to the SL, while the SQP and nonlinear

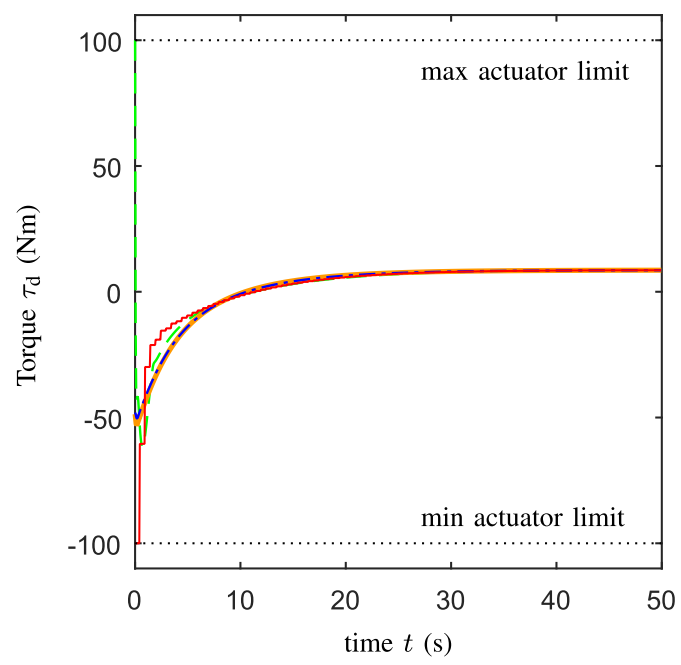

(a)

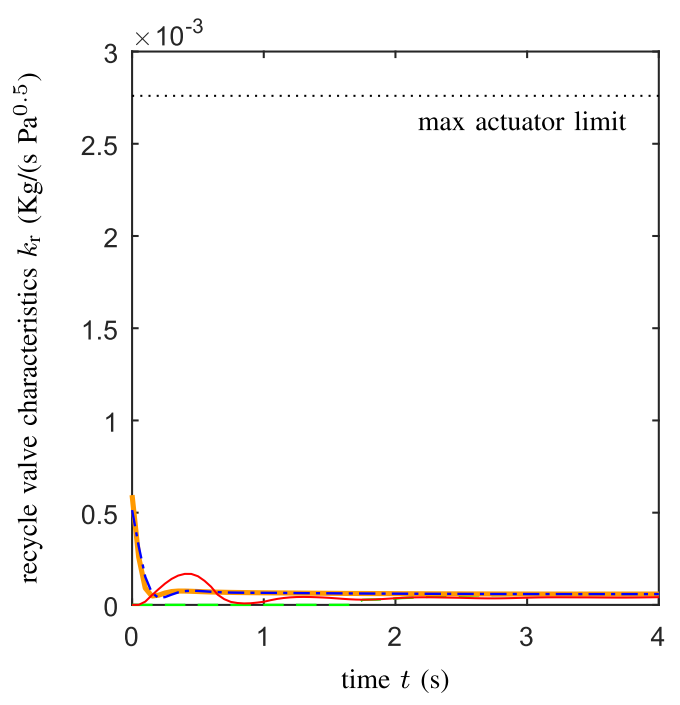

(b)

Fig. 6. Nominal case (second scenario) - Torque and recycle valve actuation, details of the first seconds of the simulation: the red line corresponds to the standard PID control, the dashed light green line corresponds to the linearized MPC, dash-dot blue to the nonlinear MPC and the orange thick to the SQP MPC.

MPC stay on the right side of the SCL by operating the recycle valve, as shown in Fig. 6(b). Again, setting $M=1$ iterations is enough to track the nonlinear MPC closely in closed loop. The differences between the linearized and nonlinear MPC are due to the more accurate dynamics employed in the prediction, and become more evident that the further the current state is from the desired target, around which the linearization matrices are computed.

\section{B. Sensitivity Analysis}

In industrial practice, the correct operation of the compression system is jeopardized by disturbances and possible model mismatch. The most critical disturbances are caused by unmodeled back pressure of the upstream and downstream processes. In fact, an increase of the outlet pressure, or equivalently a decrease of the inlet pressure, has the effect of increasing the pressure ratio, and hence acts to move the system closer to the SL. 


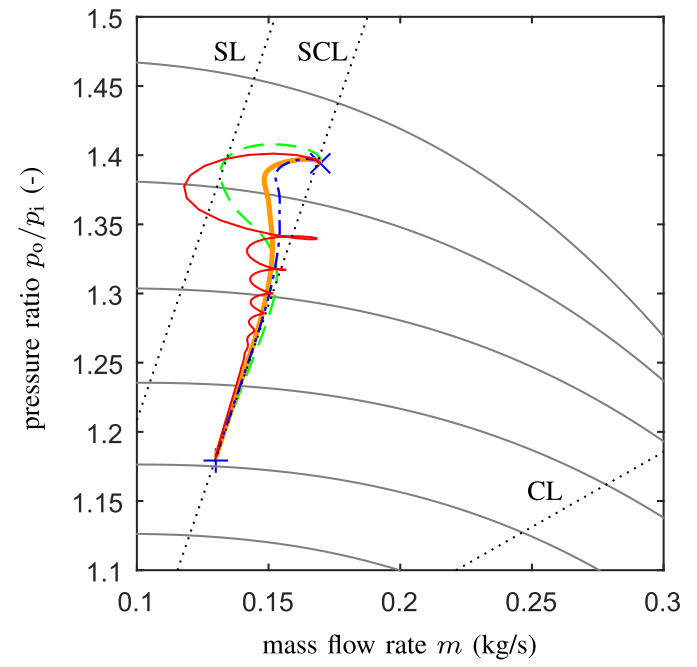

Fig. 7. Constant pressure disturbance simulation - Compressor map: the red line corresponds to the standard PID control, the dashed light green line corresponds to the linearized MPC, dash-dot blue to the nonlinear MPC and the orange thick to the SQP MPC.

Some assumptions and approximations used in the modeling phase lead typically to plant-model mismatches. For example, the nonlinear behavior of valves and the extrapolation of the compressor map to the unstable range. Moreover, degradation and maintenance introduce slow time-varying effects that are usually neglected in the modeling. In order to investigate the influence of disturbances and plant-model mismatch, a sensitivity analysis is carried out and the results are compared with the nominal simulation case.

Back pressure of the upstream/downstream process is considered by augmenting the model equations in (1) as follows:

$$
\begin{aligned}
\dot{p}_{\mathrm{i}} & =\frac{a_{\mathrm{i}}^{2}}{V_{\mathrm{i}}}\left(m_{\mathrm{i}}\left(p_{\mathrm{i}}\right)-m+m_{\mathrm{r}}\right)+d_{\mathrm{i}} \\
\dot{p}_{\mathrm{o}} & =\frac{a_{\mathrm{o}}^{2}}{V_{\mathrm{o}}}\left(-m_{\mathrm{o}}\left(p_{\mathrm{o}}\right)+m-m_{\mathrm{r}}\right)+d_{\mathrm{o}} \\
\dot{m} & =\frac{A}{L}\left(\pi(\omega, m) p_{\mathrm{i}}-p_{\mathrm{o}}\right) \\
\dot{\omega} & =\frac{1}{J}\left(\tau_{\mathrm{d}}-\tau_{\mathrm{c}}(\omega, m)-v \omega\right) \\
\dot{m}_{\mathrm{r}} & =\frac{1}{T_{\mathrm{r}}}\left(m_{\mathrm{r}, \mathrm{ss}}\left(p_{\mathrm{i}}, p_{\mathrm{o}}, k_{\mathrm{r}}\right)-m_{\mathrm{r}}\right),
\end{aligned}
$$

where $d_{\mathrm{i}}<0$ and $d_{\mathrm{o}}>0$ are constant additive process disturbances. The disturbance affects only the simulation plant model, as it is not considered in the control plant model.

The results, relative to the second scenario, are displayed graphically in Fig. 7 for $d_{\mathrm{i}}=-d_{\mathrm{o}}=-0.03 \mathrm{bar} / \mathrm{s}$. The conventional control enters the surge zone, and similarly the offset-free linearized control reaches the SL. The SQP and to a larger extent the nonlinear MPC keep closer to the safe zone, stabilizing to the desired operating point.

For a quantitative analysis of the results, the following performance metrics are introduced:

$$
\begin{aligned}
\mathrm{AIEu} & =\sum_{k}\left|u_{k}+\bar{u}\right| \\
\mathrm{RIEy} & =\sum_{k}\left|y_{k}-\bar{y}\right| \\
\mathrm{SD}_{\text {min }} & =\min _{k} \mathrm{SD}
\end{aligned}
$$

TABLE I

Performance Metrics in the Nominal Case (Benchmark)

\begin{tabular}{lrrrr}
\hline Metrics & Conventional & Lin MPC & SQP MPC & NL MPC \\
\hline nominal case & & & & \\
$\operatorname{AIEu}\left(\tau_{\mathrm{d}}\right)$ & 100.00 & 100.20 & 106.87 & $\mathbf{1 0 5 . 2 3}$ \\
$\operatorname{AIEu}\left(k_{\mathrm{r}}\right)$ & $\mathbf{1 0 0 . 0 0}$ & 101.86 & 107.94 & 107.45 \\
$\operatorname{RIEy}\left(p_{\mathrm{o}} / p_{\mathrm{i}}\right)$ & 100.00 & 108.93 & $\mathbf{9 1 . 5 1}$ & 94.96 \\
$\operatorname{RIEy}(\mathrm{SCD})$ & 100.00 & 148.46 & $\mathbf{3 6 . 7 4}$ & 47.41 \\
$\mathrm{SD}$ & 100.00 & 161.87 & $\mathbf{2 7 3 . 4 2}$ & $\mathbf{2 7 3 . 4 3}$ \\
\hline
\end{tabular}

TABLE II

Performance Metrics for Constant Pressure Disturbance

\begin{tabular}{lrrrr}
\hline Metrics & Conventional & Lin MPC & SQP MPC & NL MPC \\
\hline disturbance & $d_{\mathrm{i}}=-d_{\mathrm{o}}=-0.03 \mathrm{bar} / \mathrm{s}$ & & \\
$\mathrm{AIEu}\left(\tau_{\mathrm{d}}\right)$ & 100.36 & $\mathbf{9 7 . 0 0}$ & 104.72 & 104.16 \\
$\mathrm{AIEu}\left(k_{\mathrm{r}}\right)$ & 272.35 & $\mathbf{2 7 0 . 5 5}$ & 274.31 & 275.52 \\
$\mathrm{RIEy}\left(p_{\mathrm{o}} / p_{\mathrm{i}}\right)$ & $\mathbf{1 0 0 . 1 0}$ & 120.65 & 104.99 & 105.98 \\
$\mathrm{RIEy}(\mathrm{SCD})$ & 244.94 & 238.35 & 156.17 & $\mathbf{1 0 5 . 6 5}$ \\
$\mathrm{SD}$ & -98.02 & -6.87 & 130.64 & $\mathbf{1 6 1 . 6 9}$ \\
\hline
\end{tabular}

with $k$ spanning the simulation time and where the absolute integral error on each input, AIEu, according to (4) represents each actuator displacement, the relative integral error on each output, RIEy, integrates the distance in transient from the desired reference $\bar{y}$, and the minimum $\mathrm{SD}, \mathrm{SD}_{\min }$, is the minimum safety margin from the surge zone during the transient. Negative values of $\mathrm{SD}_{\min }$ indicate the violation of the SL constraint.

Note that more positive $\mathrm{SD}_{\min }$ values indicate more effective surge protection compared with smaller or negative values, as can also be seen in the compressors maps. Table I shows the metrics in the nominal case (second scenario) and it serves as a reference for the sensitivity analysis. Each metric is reported as a percentage of the respective metric for the conventional controller, considered as the benchmark. For instance, the minimum SD achieved by SQP and nonlinear MPC is more than double (hence safer) that obtained by the conventional controller, as can also be seen in the compressor map of Fig. 5. The best performance for every metric is highlighted in bold font.

The results for the constant pressure disturbance simulation are presented in Table II. As before, each metric is expressed as a percentage of the corresponding metric in the nominal case for the conventional controller, set to $100 \%$ in Table I. Although the controllers use the recycle valve to a similar extent [see $\operatorname{AIEu}\left(k_{\mathrm{r}}\right)$ ], they yield different performance for the SCD [see RIEy(SCD)], and minimum SD, SD min. The SQP and nonlinear MPC have SCD values [RIEy(SCD)] more than, respectively, $80 \%$ and $130 \%$ smaller, compared with the conventional controller in the same conditions. The minimum SD for the two cases, $\mathrm{SD}_{\min }$, is still around $30 \%$ and $60 \%$ better than the benchmark, in contrast to conventional control and linearized MPC both of which enter the surge region as can be seen from the negative $\mathrm{SD}_{\min }$ values. In this particular simulation, we observe the largest deviation between the proposed SQP MPC and nonlinear MPC. 
TABLE III

Performance Metrics for Model Parameter Mismatch

\begin{tabular}{|c|c|c|c|c|}
\hline Metrics & Conventional & Lin MPC & SQP MPC & NL MPC \\
\hline \multicolumn{5}{|c|}{ mismatch on dynamic parameters, $\Theta_{\mathrm{d}}, b=40 \%$} \\
\hline $\operatorname{AIEu}\left(\tau_{\mathrm{d}}\right)$ & 180.31 & 123.08 & 131.91 & 128.97 \\
\hline $\operatorname{AIEu}\left(k_{\mathrm{r}}\right)$ & 134.75 & 133.25 & 138.74 & 138.01 \\
\hline $\operatorname{RIEy}\left(p_{\mathrm{o}} / p_{\mathrm{i}}\right)$ & 111.74 & 114.58 & 97.64 & 100.99 \\
\hline RIEy(SCD) & 3952.44 & 349.10 & 142.78 & 111.50 \\
\hline $\mathrm{SD}_{\min }$ & -182.73 & 7.85 & 168.44 & 187.90 \\
\hline \multicolumn{5}{|c|}{ mismatch on valve characteristics, $\Theta_{\mathrm{v}}, b=10 \%$} \\
\hline $\operatorname{AIEu}\left(\tau_{\mathrm{d}}\right)$ & 100.15 & 99.36 & 106.26 & 104.93 \\
\hline $\operatorname{AIEu}\left(k_{\mathrm{r}}\right)$ & 164.62 & 166.51 & 170.94 & 170.95 \\
\hline $\operatorname{RIEy}\left(p_{\mathrm{o}} / p_{\mathrm{i}}\right)$ & 100.01 & 111.91 & 95.70 & 98.56 \\
\hline RIEy(SCD) & 144.06 & 205.98 & 30.63 & 21.34 \\
\hline $\mathrm{SD}_{\min }$ & 62.79 & 114.04 & 254.08 & 266.06 \\
\hline \multicolumn{5}{|c|}{ mismatch on all parameters, $\Theta_{\mathrm{d}}$ and $\Theta_{\mathrm{s}}, b=5 \%$} \\
\hline $\operatorname{AIEu}\left(\tau_{\mathrm{d}}\right)$ & 95.68 & 95.72 & 103.28 & 101.84 \\
\hline $\operatorname{AIEu}\left(k_{\mathrm{r}}\right)$ & 131.42 & 133.31 & 138.40 & 138.27 \\
\hline $\operatorname{RIEy}\left(p_{\mathrm{o}} / p_{\mathrm{i}}\right)$ & 100.18 & 110.10 & 90.46 & 93.33 \\
\hline RIEy(SCD) & 137.57 & 183.87 & 32.26 & 41.65 \\
\hline $\mathrm{SD}_{\min }$ & 36.89 & 154.96 & 146.23 & 148.21 \\
\hline
\end{tabular}

A model mismatch analysis is formulated by introducing a deterministic error on the process model parameters. The distinction is made between dynamic parameters $\Theta_{\mathrm{d}}$, defining time constants of the ODEs in (1), e.g., $V_{\mathrm{i}}, V_{\mathrm{o}}, J$, and static parameters $\Theta_{\mathrm{s}}$, comprising both the valve characteristics $\Theta_{\mathrm{v}}$ and coefficients of maps $\Theta_{\mathrm{m}}$. Thus, the sets of parameters are defined as follows:

$$
\begin{aligned}
& \Theta_{\mathrm{d}}:=\left[\begin{array}{llllllll}
a_{\mathrm{i}} & a_{\mathrm{o}} & V_{\mathrm{i}} & V_{\mathrm{o}} & A & L & J & T_{\mathrm{r}}
\end{array}\right]^{\top} \\
& \Theta_{\mathrm{s}}:=\left[\Theta_{\mathrm{v}} ; \Theta_{\mathrm{m}}\right] \\
& \Theta_{\mathrm{v}}:=\left[\begin{array}{ll}
k_{\mathrm{i}} & k_{\mathrm{o}}
\end{array}\right]^{\top} \\
& \Theta_{\mathrm{m}}:=\left[\begin{array}{ll}
\theta_{\tau} & \theta_{\pi}
\end{array}\right]^{\top},
\end{aligned}
$$

where $\theta_{\tau}$ values are the coefficients for the torque map, $\tau_{\mathrm{c}}(\omega, m)$, and $\theta_{\pi}$ the coefficients of the compressor map, $\pi(\omega, m)$. The representative perturbed element $\tilde{\theta}_{j}$ is then defined as

$$
\tilde{\theta}_{j}=\theta_{j}(1 \pm b)
$$

where $\theta_{j}$ is the corresponding parameter used for control design, and the sign of \pm is always taken in the most critical direction, e.g., it is a minus for the outlet valve characteristics, $k_{0}$, since this moves the system closer to surge. The compressor map in $\Theta_{\mathrm{m}}$ is offset and rotated, compared with the nominal case, in order to model typical interpolation errors.

Table III summarizes the results of the analysis, along with the corresponding perturbation magnitude, $b$. Three separate case studies are considered:

1) mismatch on dynamic parameters $\Theta_{d}$;

2) mismatch on valve characteristics $\Theta_{v}$;

3) mismatch on all the parameters $\Theta_{d}, \Theta_{v}$ and $\Theta_{m}$.

The results are again reported in percentage of the respective metric in the nominal case for the conventional controller.

As expected, the system is not particularly sensitive to variations of the dynamic parameters, especially for the MPC
TABLE IV

COMPUTATIONAL TIMES With FORCES PRO

\begin{tabular}{llll}
\hline Method & Avg. time $(\mathrm{ms})$ & Avg. \# iterations & Worst case $(\mathrm{ms})$ \\
\hline Linear MPC & 0.77 & 13.8 & 0.88 \\
SQP MPC & 0.84 & 14.3 & 1.07 \\
Nonlinear MPC & 0.96 & 13.3 & 1.07 \\
\hline
\end{tabular}

controllers. A factor $b$ of $40 \%$ on every dynamic parameter is considered, with the sign in (30) arranged such that the plant response is slower than what expected. The benefits of the SQP and nonlinear MPC in terms of minimum SD, $\mathrm{SD}_{\min }$, are maintained, as it is still at least $60 \%$ larger than the benchmark case. The conventional control enters surge, affecting the error on the SCD, i.e., high RIEy(SCD) and negative $\mathrm{SD}_{\min }$, while the linearized MPC yields a poor SD margin. It is also interesting to note that the SQP and nonlinear MPC use the torque actuation comparably to the linearized MPC, as can be seen from the metric $\operatorname{AIEu}\left(\tau_{\mathrm{d}}\right)$, while achieving a smaller error $\operatorname{RIEy}\left(p_{\mathrm{o}} / p_{\mathrm{i}}\right)$.

For variations of the valve characteristics, the changes are more noticeable. For a factor $b=10 \%$, all the controllers reach the desired operating point. The controllers use comparably the recycle valve actuation $\left[\right.$ see $\left.\operatorname{AIEu}\left(k_{\mathrm{r}}\right)\right]$, but the error on the SCD, RIEy(SCD), is significantly larger for the conventional control and the linear MPC than for the SQP and nonlinear MPC. The latter controllers achieve a minimum $\mathrm{SD}, \mathrm{SD}_{\min }$, that is only slightly deteriorated, compared with the nominal case, in contrast to the conventional controller and the linearized MPC, for which the corresponding reduction in margin are around $40 \%$.

Finally, for a combined mismatch of all the parameters, the considered deviation factor is $b=5 \%$. For a fixed speed and mass flow rate, the actual compressor map has a lower pressure ratio than the nominal case, and similarly for the torque map. With this setting, all the MPC techniques achieve a sufficient minimum SD with relatively small recycle valve actuation, $\operatorname{AIEu}\left(k_{\mathrm{r}}\right)$. The conventional control does not enter surge, but it approaches dangerously close to the SL. As in the other case, the SQP and nonlinear MPC obtain a similar error on the SCD [RIEy(SCD)], compared with the other techniques. They track the SCL very accurately during the transient. Apart from this metric, in this configuration, the linearized MPC performs similar to the SQP and nonlinear MPC.

Also in this sensitivity analysis, we remark that the SQP MPC with $M=1$ closely approximates the nonlinear MPC in terms of the computed metrics. Moreover, using RK4 instead of Euler discretization yields minor differences in terms of closed-loop performance that are most noticeable in the constant pressure simulation.

\section{Computational Times}

The computation times for the linear MPC, SQP MPC, and nonlinear MPC are given in Table IV. The time comprises both the preparation of the problem, i.e., building the Jacobian matrices, and solving the optimization problem. The calculations have been performed in $\mathrm{C}$, both on an industrial Programmable Logic Controller (PLC) ABB AC 800PEC, 
TABLE V

COMPRESSION SYSTEM SPECIFICATIONS

\begin{tabular}{ll}
\hline Specification & Numerical value \\
\hline Electrical Drive Power & $15 \mathrm{~kW}$ \\
Torque Limitation & {$[-100,100] \mathrm{Nm}$} \\
Inlet Tank Volume & $V_{\mathrm{i}}=1.172 \mathrm{~m}^{3}$ \\
Outlet Tank Volume & $V_{\mathrm{o}}=0.595 \mathrm{~m}^{3}$ \\
Duct Ratio & $\frac{A}{L}=1.676 \mathrm{e}-4 \mathrm{~m}$ \\
Rotational Inertia & $J=2 \mathrm{~kg} \mathrm{~m}^{2}$ \\
Recycle Valve Constant & $T_{\mathrm{r}}=0.5 \mathrm{~s}$ \\
\hline
\end{tabular}

with control unit PowerPC 800-MHZ dual-core PP D539 (only one core is dedicated to the optimization solver). It is interesting to notice that thanks to the fast solver FORCES PRO [32], all of the MPC problems can be solved on average in milliseconds, with worst case computational times by far below the chosen sampling time $\left(T_{\mathrm{S}}=50 \mathrm{~ms}\right)$. The QPs in the linear and SQP MPC are solved by the Mehrotra predictorcorrector interior-point method. The NLP solver finds a KKT point of the NLP by using the barrier interior-point method.

\section{CONCLUSION}

We have discussed MPC approaches for centrifugal compression systems, with nonlinear model dynamics, controlled by torque and recycle valve actuation. Such systems are typically employed in critical processes, where the compressed fluid can damage the compressor irreversibly.

We have first considered the contractive MPC formulation to ensure closed-loop stability for the nominal model dynamics, and we have proposed a numerical procedure for testing recursive feasibility over an ellipsoidal domain of attraction.

Since the compression system considered is typically subject to unmodeled dynamics and disturbance inputs, we have discussed the application of linear and nonlinear offset-free MPC. From the computational perspective, in the nonlinear case, we have also considered the SQP approach. In our numerical experience, one iteration, also called real-time iteration, yields good closed-loop performance, comparable to a full nonlinear solution.

As closed-loop stability for the full nonlinear, perturbed model is hard to establish in a numerically tractable manner, we have tested in simulation all the MPC approaches discussed and compared them with the standard industrial control, which consists of PI regulators with antiwindup corrections. Our numerical simulations include sensitivity tests to typical exogenous disturbances and model mismatch for all the controllers.

We conclude that MPC approaches are beneficial in improving the performance of standard industrial controllers for active surge regulation. In particular, the SQP and nonlinear MPC approaches are capable of keeping the system at a safe distance from the surge zone in all the cases tested, in particular in the presence of inlet/outlet pressure disturbances and for mismatch on the dynamic parameters. From our computational experience, we conclude that the real-time implementation is feasible for all MPC approaches. It follows that the SQP and nonlinear MPC are the leading candidates for industrial deployment.
Future work includes the experimental validation of these MPC approaches on the actual industrial system.

\section{APPENDIX \\ COMPRESSOR SPECIFICATIONS}

The centrifugal compressor used for the simulation is a Continental 020, whose specifications are given in Table V, together with the compression system details.

\section{ACKNOWLEDGMENT}

The authors would like to thank T. Robbiani (ETH Zurich) and A. Domahidi, J. L. Jerez, and A. Hempel (Embotech) for their help in the implementation of the controllers in C.

\section{REFERENCES}

[1] J. O. Lamell, T. Trumbo, and T. F. Nestli, "Offshore platform powered with new electrical motor drive system," in Proc. Ind. Appl. Soc. 52nd Annu. Petroleum Chem. Ind. Conf., Sep. 2005, pp. 259-266.

[2] G. Torrisi, S. Mariéthoz, R. S. Smith, and M. Morari, "Comparison of the efficiency of different magnetization strategies for a variable speed induction machine drive," in Proc. IEEE Eur. Conf. Power Electron. Appl., 2015, pp. 1-10.

[3] F. McCaughan, "Bifurcation analysis of axial flow compressor stability," SIAM J. Appl. Math., vol. 50, no. 5, pp. 1232-1253, 1990.

[4] C. Hõs, A. Champneys, and L. Kullmann, "Bifurcation analysis of surge and rotating stall in the Moore-Greitzer compression system," IMA J. Appl. Math., vol. 68, no. 2, pp. 205-228, 2003.

[5] A. Agarwal and N. Ananthkrishnan, "Bifurcation analysis for onset and cessation of surge in axial flow compressors," Int. J. Turbo Jet Engines, vol. 1, no. 17, pp. 207-217, 2000.

[6] E. H. Abed, P. K. Houpt, and W. M. Hosny, "Bifurcation analysis of surge and rotating stall in axial flow compressors," J. Turbomach. vol. 115, no. 4, pp. 817-824, 1993.

[7] N. Ananthkrishnan, U. Vaidya, and V. Walimbe, "Global stability and control analysis of axial compressor stall and surge phenomena using bifurcation methods," Proc. Inst. Mech. Eng. A, J. Power Energy, vol. 217, no. 3, pp. 279-286, 2003.

[8] E. M. Greitzer, "Surge and rotating stall in axial flow compressorsPart I: Theoretical compression system model," J. Eng. Power, vol. 98, no. 2, pp. 190-198, 1976.

[9] E. M. Greitzer, "Surge and rotating stall in axial flow compressorsPart II: Experimental results and comparison with theory," J. Eng. Power, vol. 98, no. 2, pp. 199-211, 1976.

[10] J. T. Gravdahl, O. Egeland, and S. O. Vatland, "Drive torque actuation in active surge control of centrifugal compressors," Automatica, vol. 38 , no. 11 , pp. 1881-1893, 2002.

[11] M. Krstic, D. Fontaine, P. Kokotović, and J. D. Paduano, "Useful nonlinearities and global stabilization of bifurcations in a model of jet engine surge and stall," IEEE Trans. Autom. Control, vol. 43, no. 12, pp. 1739-1745, Dec. 1998.

[12] J. T. Gravdahl and O. Egeland, "Centrifugal compressor surge and speed control," IEEE Trans. Control Syst. Technol., vol. 7, no. 5, pp. 567-579, Sep. 1999.

[13] B. Bohagen and J. T. Gravdahl, "Active control of compression systems using drive torque; a backstepping approach," in Proc. IEEE Conf. Decision Control, Eur. Control Conf. (CDC-ECC), Dec. 2005, pp. 2493-2498.

[14] J. S. Simon and L. Valavani, "A Lyapunov based nonlinear control scheme for stabilizing a basic compression system using a close-coupled control valve," in Proc. IEEE Amer. Control Conf., 1991, pp. 2398-2406.

[15] N. Staroselsky and L. Ladin, "More effective control for centrifugal gas compressors operating in parallel," in Proc. ASME Int. Gas Turbine Conf. Exhibit, 1986, pp. 1-12.

[16] N. Branley Shaun and K. Narayanan, "A total integrated approach," Conoco Phillips, Australia PTY LTD, Tech. Rep., West Perth, WA, Australia, 2012. [Online]. Available: https://www.cccglobal.com/a-totalintegrated-approach/

[17] N. Dukle and K. Narayanan, "Validating anti-surge control systems," Petroleum Technol. Quart., vol. 8, pp. 87-96, Summer 2003. 
[18] T. Bentaleb, A. Cacitti, S. De Franciscis, and A. Garulli, "Multivariable control for regulating high pressure centrifugal compressor with variable speed and IGV," in Proc. IEEE Conf. Control Appl., Oct. 2014, pp. 486-491.

[19] T. Bentaleb, A. Cacitti, S. De Franciscis, and A. Garulli, "Model predictive control for pressure regulation and surge prevention in centrifugal compressors," in Proc. IEEE Eur. Control Conf., Jul. 2015, pp. 3351-3356.

[20] S. Budinis and N. Thornhill, "Control of centrifugal compressors via model predictive control for enhanced oil recovery applications," IFACPapersOnLine, vol. 48, no. 6, pp. 9-14, 2015.

[21] A. Cortinovis, D. Pareschi, M. Mercangoez, and T. Besselmann, "Model predictive anti-surge control of centrifugal compressors with variablespeed drives," in Proc. IFAC Workshop Autom. Control Offshore Oil Gas Prod., 2012, pp. 251-256.

[22] A. Cortinovis, H. J. Ferreau, D. Lewandowski, and M. Mercangöz, "Safe and efficient operation of centrifugal compressors using linearized MPC," in Proc. IEEE Conf. Decision Control, Dec. 2014, pp. 3982-3987.

[23] A. Cortinovis, H. Ferreau, D. Lewandowski, and M. Mercangöz, "Experimental evaluation of MPC-based anti-surge and process control for electric driven centrifugal gas compressors," J. Process Control, vol. 34, pp. 13-25, Oct. 2015.

[24] T. A. Johansen, "On multi-parametric nonlinear programming and explicit nonlinear model predictive control," in Proc. IEEE Conf. Decision Control, vol. 3. Dec. 2002, pp. 2768-2773.

[25] G. Torrisi, V. Jaramillo, J. R. Ottewill, S. Mariéthoz, M. Morari, and R. S. Smith, "Active surge control of electrically driven centrifugal compressors," in Proc. IEEE Eur. Control Conf., Jul. 2015, pp. 1608-1613.

[26] G. Torrisi, S. Grammatico, M. Morari, and R. S. Smith, "Model predictive control approaches for centrifugal compression systems," in Proc. IEEE Conf. Decision Control, Dec. 2015, pp. 4320-4325.

[27] S. L. D. O. Kothare and M. Morari, "Contractive model predictive control for constrained nonlinear systems," IEEE Trans. Autom. Control, vol. 45, no. 6, pp. 1053-1071, Jun. 2000.

[28] M. J. Tenny and S. J. Wright, "A feasible trust-region sequential quadratic programming algorithm," SIAM J. Optim., vol. 4, no. 4, pp. 490-530, 2005.

[29] M. J. Tenny, S. J. Wright, and J. B. Rawlings, "Nonlinear model predictive control via feasibility-perturbed sequential quadratic programming," Comput. Optim. Appl., vol. 28, no. 1, pp. 87-121, 2004.
[30] M. Diehl, H. Bock, J. Schlöder, R. Findeisen, Z. Nagy, and F. Allgöwer, "Real-time optimization and nonlinear model predictive control of processes governed by differential-algebraic equations," J. Process Control, vol. 12, no. 4, pp. 577-585, 2002.

[31] M. Diehl, R. Findeisen, F. Allgower, H. G. Bock, and J. P. Schlöder, "Nominal stability of real-time iteration scheme for nonlinear mode predictive control," IEE Proc.-Control Theory Appl., vol. 152, no. 3, pp. 296-308, 2005.

[32] A. Domahidi and J. Jerez. (Jul. 2014). FORCES professional. embotech GmbH. [Online]. Available: http://embotech.com/FORCES-Pro

[33] D. Q. Mayne, J. B. Rawlings, C. V. Rao, and P. O. M. Scokaert, "Constrained model predictive control: Stability and optimality," Automatica, vol. 36, no. 6, pp. 789-814, 2000

[34] G. Pannocchia and J. B. Rawlings, "Disturbance models for offset-free model-predictive control," AIChE J., vol. 49, no. 2, pp. 426-437, 2003

[35] U. Maeder, F. Borrelli, and M. Morari, "Linear offset-free model predictive control," Automatica, vol. 45, no. 10, pp. 2214-2222, 2009.

[36] M. Morari and U. Maeder, "Nonlinear offset-free model predictive control," Automatica, vol. 48, no. 9, pp. 2059-2067, 2012.

[37] J. Gravdahl, O. Egeland, and S. Vatland, "Active surge control of centrifugal compressors using drive torque," in Proc. IEEE Conf. Decision Control, vol. 2. Dec. 2001, pp. 1286-1291.

[38] B. W. Batson, "Invariant coordinate systems for compressor control," in Proc. ASME Int. Gas Turbine Aeroengine Congr. Exhibit., 1996, pp. 1-16.

[39] B. Bøhagen and J. Gravdahl, "Active surge control of compression system using drive torque," Automatica, vol. 44, no. 4, pp. 1135-1140, 2008.

[40] G. Quartarone, N. Anglani, and S. Riverso, "Model predictive control: First application of a novel control strategy for adjustable speed drive compressors," in Proc. Conf. IEEE Ind. Electron. Soc. (IECON), Nov. 2013, pp. 7892-7897.

[41] G. Torrisi, S. Grammatico, R. S. Smith, and M. Morari. (2016). "A projected gradient and constraint linearization method for nonlinear model predictive control." [Online]. Available: https://arxiv.org/abs/1610.06834 